\title{
Relación universidad-institución cultural: eje para la formación de una cultura laboral en estudiantes
}

\author{
Relationship University-Cultural Institution: \\ Axis for the Formation of a Work Culture in Students
}

\author{
Camen Velázquez Quintana \\ Dagneris Batista de los Ríos \\ 23984@ltu.sld.cu dagneris@ltu.sld.cu \\ https://orcid.org/0000-0002-9876-9162 https://orc id.org/0000-0002-1005-4876 \\ Universidad de LasTunas| Cuba
}

\begin{abstract}
Resumen
La relación entre la universidad y las instituciones culturales constituye el eje articulador para la realización de la práctica laboral o preprofesional de los estudiantes universitarios. La labor educativa que realiza la Biblioteca Provincial en la gestión de estas prácticas prepara a los estudiantes para que sean capaces de dar respuesta a los problemas que se presentan en su quehacer profesional, lo que se materializa en la formación de una cultura laboral que favorece la modelación de actitudes, de comportamientos y la transformación de los contextos donde interactúan, lo que tributa al desarrollo social.
\end{abstract}

\section{Palabras clave}

cultura laboral, práctica laboral, desarrollo social, estudiantes universitarios

\begin{abstract}
The relationship between the university and the cultural institutions constitute the articulating axis for the realization of the professional or pre-professional practice of the university students. The educational work carried out by the Provincial Library in the management of these practices prepares students to be able to respond to problems that arise in their professional work, which materializes in the formation of a work culture, that favors the modeling of attitudes, of behaviors and the transformation of the contexts in which it interacts, which taxes social development.
\end{abstract}


En la relación sociedad-cultura, los objetivos se direccionan a la preservación y al desarrollo de la cultura, para lograr la transformación de los sujetos y de las comunidades. El cumplimiento de este encargo social no corresponde a una institución específica, sino a toda la sociedad, por la necesidad de evolucionar hacia niveles superiores del conocimiento humano y de transformar el medio. En este sentido se han encaminado los esfuerzos tendientes a fortalecer la relación que se establece entre la universidad y las instituciones culturales del territorio, ya que ambas desde su misión favorecen la formación integral de los estudiantes como agentes activos del conocimiento, capaces de transformar su realidad social en los diferentes contextos de actuación y, por ende, de lograr su propia transformación.

No se trata de ejecutar acciones de manera homogénea, sino de analizar en cada institución cuáles son las que favorecen la formación integral del estudiante cuando éste desarrolla su práctica laboral o preprofesional, intencionadas a la creación de valores culturales, a la conservación de los ya creados, a la divulgación y la formación de una cultura laboral. El ciclo de las actividades de desarrollo y de transformación cultural significa la solución del problema, la satisfacción de la necesidad, en aras de potenciar el desarrollo social.

La experiencia se realiza en la Biblioteca Provincial José Martí con los estudiantes que realizan su práctica laboral en esta entidad. A partir de un diagnóstico que se les aplica, se conoce cuáles son sus limitaciones, sus fortalezas, sus motivaciones con la profesión, entre otros aspectos; información que les permite a los tutores que se les asignan en la institución diseñar acciones para que cada estudiante se convierta en protagonista al superar sus deficiencias.

Se utilizaron los métodos análisis y crítica de las fuentes, análisis-síntesis, lógico-histórico, observación participante, entrevistas y la triangulación de datos. De manera general, se trabaja para formar en los estudiantes una cultura laboral que les permita responder a las problemáticas profesionales y sociales una vez graduados, por ello, es muy importante no realizar acciones en abstracto, sino sobre la base de los problemas del contexto cultural formativo, que modelen sus actitudes y sus comportamientos. 


\section{Desamollo}

La cultura se concibe como un aspecto fundamental en la comprensión del ser humano y de la sociedad. Se forma a partir de características particulares, manifestándose en los aspectos materiales, espirituales y afectivos como motor de su desarrollo, lo cual determina la calidad de vida de la sociedad.

La UNESCO (2001) establece que «la cultura es el conjunto de rasgos distintivos, espirituales, materiales, intelectuales, afectivos, que caracterizan una sociedad o grupos sociales. Engloba además de las artes y las letras, los modos de vida, los derechos fundamentales del ser humano, los sistemas de valores, las tradiciones y las creencias» (en línea). La trascendencia de la cultura se reconoce a partir de lo que el hombre ha creado y de su compromiso con el desarrollo social y político basado en sus propias realizaciones, para lo cual crea un sistema con múltiples funciones que se enriquecen a partir del acelerado desarrollo social y científico tecnológico.

En el siglo XXI, la universidad reclama las exigencias de una sólida formación cultural como fundamento de la comprensión global de la época. Le corresponde ser un factor clave para el desarrollo científico; el modelo de universidad científica, tecnológica y humanista conjuga fortalezas que son atributos únicos: una elevada concentración de hombres de ciencia y de pensamiento que hace de la universidad una institución social con capacidad de generar nuevos conocimientos y habilidades y de socializar dichos conocimientos.

La universidad alcanza un mayor impacto en la sociedad cuando responde a las necesidades actuales de cada territorio y de cada comunidad. Para ello, se establecen convenios con instituciones que, por su objeto social, tienen relación con determinadas carreras y están en condiciones de preparar a los estudiantes para acercarlos a problemas reales que enfrentarán una vez graduados; fomentan el amor por la profesión y generan un sentido de pertinencia, ya que estas instituciones pueden ser receptoras de estos futuros profesionales que han contribuido a formar.

Las instituciones culturales son las encargadas de ampliar y de profundizar, mediante la promoción y la investigación, la herencia cultural, de trasmitir las actitudes que asumen los hombres para conservar, defender, promover los bienes y valores de su patrimonio, y de establecer procesos de comunicación que enriquecen el nivel de desarrollo al convertir a los sujetos en portadores y en receptores de información que, al ser seleccionada con criterios educativos, eleva la cultura general de los sujetos. 
La Universidad de Las Tunas, mediante convenios realizados con la Dirección Provincial de Cultura y su red de instituciones culturales, favorece la realización de prácticas laborales de estudiantes de diferentes carreras. Un ejemplo de estas instituciones es la Biblioteca Provincial José Martí, ubicada en el municipio cabecera de la provincia, la cual recibe estudiantes de 2do a 5to año de las carreras de Comunicación Social y de Gestión Sociocultural para el Desarrollo.

$\mathrm{Al}$ iniciar las prácticas laborales, que responden a una asignatura, los estudiantes recibían una guía con objetivos definidos y en la entidad se les daba a conocer el banco de problemas identificados y las tareas a cumplir. Sin embargo, no se alcanzaban los resultados esperados y se limitaba la capacidad científica de los estudiantes de investigar. Por ello, se proyectó un cambio en la concepción de la práctica laboral de conjunto entre los tutores de la universidad y aquellos que se designaban en la Biblioteca Provincial.

Los estudiantes debían identificar las problemáticas y proyectar las respuestas mediante acciones en la institución y en las comunidades donde se realizan las actividades extensionistas. En la propuesta se tuvo en cuenta la aplicación de un diagnóstico, lo que les permitió a los tutores asignados en la Biblioteca conocer las limitaciones o las deficiencias de los estudiantes, sus intereses y sus objetivos. En este aspecto, las principales limitaciones estaban dadas en la proyección escénica, el uso inadecuado de la lengua materna, la poca motivación por la carrera y el insuficiente gusto estético.

Se proyectaron acciones para elevar su desarrollo y para que la práctica realmente respondiera a los objetivos formativos. Se tomó como muestra diez estudiantes: cinco de la carrera de Comunicación Social, de 3er año, y cinco de Gestión Sociocultural para el Desarrollo, de 2do año. A partir del trabajo personalizado con los estudiantes, la asignación de tareas con las que debían cumplir, la exigencia por el cumplimiento del horario, la estimulación a la creación, el tratamiento adecuado, la motivación por la lectura y la búsqueda de información de diversos temas, se fueron modelando actitudes y comportamientos y los estudiantes comenzaron a conducirse de forma adecuada en los diferentes espacios, incluso, en su forma de vestir, además de aportar ideas y de gestionar actividades, lo que los hizo elevar su sentido de la responsabilidad. 
En las entrevistas realizadas expresaron con cuales actividades caracterizadoras de la entidad se sentían más identificados después de haberlas gestionado (Tabla 1).

\begin{tabular}{|c|c|c|}
\hline Lugar & Actividades & Porcentaje \\
\hline 1 & $\begin{array}{c}\text { Actividad caracterizadora organizada } \\
\text { por la Sala General y Literatura }\end{array}$ & 95.6 \\
\hline 2 & Actividades de proyectos en comunidades & 91.3 \\
\hline 3 & Actividades en centros educacionales & 89.6 \\
\hline 4 & $\begin{array}{c}\text { Actividades en organismos y en instituciones } \\
\text { como ACLIFIM, ANSOC, ANCI }\end{array}$ & 87.2 \\
\hline
\end{tabular}

Tabla 1 | Actividades organizadas por estudiantes en la entidad durante la práctica laboral (Fuente: elaboración propia)

El resultado fue novedoso. Los estudiantes no solo debían autogestionar la información, sino aportar ideas que fueran motivadoras, según el público a las que estaban dirigidas, promocionarlas y divulgarlas por los medios de comunicación, gestionar con las instituciones cercanas la presencia del auditorio y, de igual forma, en las comunidades, lo que favoreció su nivel de independencia y de creatividad. Asimismo, se elevó el impacto de las actividades, evidente en el grado de satisfacción del público y, por ende, el reconocimiento social de los estudiantes, por aportar ideas valiosas que modificaron la dinámica de trabajo de la institución.

Las actividades se caracterizaron por una dinámica que se corresponde con la dinámica de la sociedad cubana, que tiene entre sus aspiraciones lograr un mayor desarrollo humano. Se requiere, tanto en su concepción como en su ejecución, un mayor nivel de preparación teórica y práctica para, realmente, alcanzar el impacto que es necesario en la transformación de los sujetos y de los contextos, de ahí la importancia de la práctica laboral, la cual amplía la vía de comunicación en los dos sentidos: universidad-sociedad y viceversa. El resultado de esta comunicación en doble sentido se interpreta como el grado de importancia o de significación que se alcanza como resultado de la relación de la necesidad social.

La práctica laboral alcanza un mayor impacto cuando el objeto es la sociedad (cómo se influye en el medio) y de pertinencia y si el medio influye en ésta (cómo se refleja en los estudiantes para prepararlos para dar respuesta a las necesidades sociales). Las instituciones culturales —en estrecha relación con la universidad - son un eje transversal 
para el desarrollo social y para la formación de una cultura laboral en los futuros profesionales, por ello, se puede aseverar que su dirección consciente y eficiente solo es posible cuando se expresa esa relación función-proceso y, en tal sentido, se organiza su gestión.

Otro elemento medular fue la constante superación de los trabajadores de la entidad, aún cuando son designados tutores en la Biblioteca Provincial para conducir las prácticas laborales y para acompañar al estudiante. El colectivo de trabajadores desempeña una función educativa, por lo que deben estar preparados para contribuir a modelar actitudes y comportamientos en los estudiantes de acuerdo al código de trabajo de la institución. Para lograrlo, se proyectaron cursos de diferentes temáticas que favorecieran su superación, la calidad del trabajo y el apoyo necesario a los estudiantes de práctica.

Las prácticas laborales que se realizaron en la Biblioteca Provincial se enriquecieron con las actividades propuestas y gestionadas por los estudiantes como protagonistas del proceso, las cuales tuvieron un carácter educativo, axiológico, político, cultural y comunicacional. Los estudiantes se apropiaron de contenidos socialmente significativos, de experiencias y de vivencias, se estimuló la autogestión del conocimiento, el interés hacia el contenido de la profesión y el desarrollo de su personalidad.

Se favoreció, también, la construcción de significados en el contexto de relaciones humanas y en la solución de problemas que se presentan en el ámbito laboral. Los estudiantes se prepararon laboralmente en, desde y para el trabajo, por lo que fueron capaces de responder a los problemas profesionales identificados con respuestas científicas y viables, hecho que demostró los niveles de desarrollo alcanzados. Se modelaron actitudes y comportamientos en los estudiantes, expresados en la forma de conducirse, se elevó su sentido de pertinencia con la institución y la formación de valores como la responsabilidad y la solidaridad mediante acciones que transformaron el entorno social de manera flexible y creadora, desde una perspectiva humanista.

Todos estos elementos concatenados entre sí favorecieron en los estudiantes la formación de una cultura laboral, caracterizada por un conjunto de principios y de valores que los conducen a realizar actividades en el ámbito laboral, a lograr un mejor desempeño, al cumplimiento de reglas y a la modelación de actitudes y de conductas. Expresada por medio del modo de actuar y de pensar en condiciones determinadas, en las que el estudiante vincula lo aprendido desde la teoría, lo sistematiza con la investigación y lo pone en práctica, avalando su desempeño laboral. 
La cultura laboral es un proceso de transmisión, de aprehensión y de difusión de la cultura, para formar integralmente a los estudiantes desde la actividad laboral. Tiene carácter continuo en la creación de valores materiales y espirituales, un modo innovador de producir, de aplicarlos a la vida y de consolidarlos en el contexto sociolaboral para desarrollar y para transformar la sociedad. Ello dota al estudiante de independencia, de creatividad y de valores éticos, es un proceso dirigido a la transformación del estudiante en los ámbitos de formación para alcanzar los objetivos profesionales en contextos reales de actuación profesional.

La formación laboral y la cultura laboral son conceptos muy interrelacionados, el primero está dirigido al proceso que se desarrolla para obtener como resultado el segundo. Según Antonio Fong (2005), la formación laboral es el proceso de trasmisión y de adquisición de los estudiantes, el conjunto de conocimientos, de habilidades, de procedimientos y de estrategias que se necesitan para analizar, para comprender y para dar solución a los problemas de la práctica social, que están encaminados a potenciar el saber hacer y el cómo hacerlo, orientado por el sistema de valores adquirido curricularmente y en la experiencia cotidiana.

Esta formación laboral tiene por finalidad socializar mediante el trabajo, educar normas morales de conducta ante el trabajo, en la que se concentran valores como la honradez, la honestidad, el patriotismo, la responsabilidad, la laboriosidad, el colectivismo y la solidaridad, entre otros. Las capacidades, las cualidades, la cultura laboral del individuo no se heredan, sino que estos componentes de la personalidad se educan, se forman en el proceso de la actividad y de la comunicación con otras personas.

La cultura laboral es la preparación o la educación que adquiere el individuo en la esfera del trabajo en su sentido más amplio, que incluye el desarrollo de habilidades, de conocimientos y de actitudes del mundo laboral. Expresa el conjunto de conocimientos, de habilidades y de valores que conforman una concepción acerca del lugar y del papel del trabajo para el hombre y para el desarrollo social, así como una actitud consecuente ante el trabajo y el dominio de los requerimientos para su desempeño, resultado que se obtiene como parte de la formación laboral.

José Ángel Espinosa (2008) expresa: «La cultura laboral en el contexto sociocultural laboral es la expresión de la vinculación y de la aplicación de lo adquirido o construido en la universidad desde el punto de vista académico e investigativo, es la propia formación 
académica» (p. 62). La cultura laboral no debe atender solo a la actividad laboral propia, sino a una cultura profesional que constituye un modo de actuar y de pensar específicos, de acuerdo a condiciones determinadas.

También puede pensarse a esta cultura laboral como:

[...] un proceso de producción de significados en el contexto de relaciones humanas, integración de contenidos, solución a problemas que se presentan en la vida cotidiana y la transformación del entorno social de manera flexible y creadora, a partir de asumir los cambios en el universo laboral desde una perspectiva humanista, con la preparación en, desde y para el trabajo (Pérez, 2009, p. 41).

Tiene un carácter continuo en la creación de valores materiales y espirituales, un modo innovador de producirlos, de aplicarlos a la vida y de afirmarlos en el contexto sociolaboral para transformar la sociedad.

Las unidades docentes y las instituciones donde los estudiantes universitarios realizan su práctica laboral tienen una responsabilidad social importante. Estos centros deben favorecer la formación de los futuros profesionales, vista la cultura laboral como el conjunto de principios y de valores con los que nos conducimos, el desempeño en el trabajo, el cumplimiento de reglas en los contextos de actuación profesional, los principios y los valores que definen actitudes y conductas.

En cada uno de los contextos con los que interactúa, el estudiante lleva a cabo procesos de socialización y de individualización que no solo están vinculados al trabajo, sino que son también culturales por la información que aportan. De hecho, son formativos, ya que el estudiante adquiere una cultura laboral y, a su vez, profesional, al favorecerse la reflexión, la revisión y la proyección crítica de los vínculos de la teoría y la práctica, así como la relación de lo vivencial, lo empírico y lo investigado.

Según Dagneris Batista de los Ríos (2017):

[...] la cultural laboral profesional es un proceso que modela la relación entre los problemas del contexto cultural laboral formativo y el modo de actuación, su base son los problemas profesionales de cada contexto de su práctica y el modo de actuación, el cual evidencia el conocimiento de su profesión, habilidades, valores, aptitudes, métodos y procedimientos del ejercicio de la profesión (s/p). 
Le corresponde a las instituciones donde se realiza la práctica evaluar el desempeño profesional del estudiante en la solución práctica e intelectual de los problemas, el nivel de respuestas científicas y viables que generen una transformación del contexto y de los sujetos que participan en el proceso.

Resulta imprescindible para la formación que las instituciones -en conjunto con la universidad - no proyecten acciones sin tener en cuenta el diagnóstico de los estudiantes. Juega un rol esencial el colectivo de trabajadores en la conducción de este proceso, desde el ejemplo personal, la ética, el cumplimiento de la disciplina laboral, el sentido de pertenencia y el amor por su institución.

Los tutores de la universidad no pueden dejar de atender este proceso, aun cuando estén presentes los de la entidad. Esta relación es la que fortalece la realización con calidad de la práctica laboral, que no es un proceso rígido que no admite modificaciones, sino que, por el contrario, necesita alcanzar un resultado que no se logra en todos los estudiantes de igual manera. Se les debe dar la oportunidad de proyectar y de gestionar acciones educativas, axiológicas, culturales, políticas, como un trabajador de la institución según sus potencialidades, ya sea dentro de la institución o en las comunidades, donde lo más importante sea transformar una problemática identificada mediante la información y la cultura.

\section{Conclusiones}

Para garantizar la formación de una cultura laboral debe entenderse el aprendizaje como un proceso de formulación y de solución de problemas vinculados a la vida social y productiva, donde la universidad - junto con las unidades docentes y las diferentes instituciones culturales donde los estudiantes realizan la práctica laboral o preprofesional- garantizan que los estudiantes sean capaces de dar respuesta a los problemas profesionales o sociales identificados.

En la Biblioteca Provincial José Martí se gestionó el proceso a partir de un diagnóstico que se le aplica a los estudiantes, con el objetivo de que los tutores de la institución diseñaran acciones que no solo tributaron a la modelación de actitudes y de comportamientos sino que contribuyeron a elevar su desarrollo social, a lograr un mayor protagonismo, un sentido de pertinencia y la formación de una cultura laboral que se traduce en una mayor preparación y desempeño de los estudiantes. 


\section{Referencias}

BATISTA DE LOS RÍOS, D. (2017). Gestión pedagógica de la extensión universitaria para la formación integral del estudiante [Tesis de Doctorado]. Las Tunas, Cuba: Universidad de Las Tunas.

ESPINOSA, J. A. (2008). Gestión de la cultura profesional en la educación superior [Tesis de Doctorado]. Holguín, Cuba: Instituto Superior Pedagógico «José de la Luz y Caballero».

FONG, A. (2005). La formación laboral del educando en las transformaciones de la escuela básica cubana. La Habana, Cuba: Pedagogía.

PÉREZ, A. (2009). La cultura laboral en las condiciones del modelo de Secundaria Básica [Tesis de Doctorado]. Santiago de Cuba, Cuba.

UNESCO (2001). Declaración Universal sobre la Diversidad Cultural.

Recuperado de http://portal.unesco.org/es/ev.php-

URL_ID=13179\&URL_DO=DO_TOPIC\&URL_SECTION=201.html 